Check for updates

Cite this: Phys. Chem. Chem. Phys., 2021, 23, 2885

Received 10th August 2020

Accepted 8th January 2021

DOI: $10.1039 / \mathrm{d} 0 \mathrm{cp} 04236 \mathrm{f}$

rsc.li/pccp

\title{
Multi-phonon proton transfer pathway in a molecular organic ferroelectric crystal $\dagger$
}

\begin{abstract}
Matthew T. O. Okenyi, (DD ${ }^{a}$ Laura E. Ratcliff*a and Aron Walsh (D) *ab
While the majority of ferroelectrics research has been focused on inorganic ceramics, molecular ferroelectrics can also combine large spontaneous polarization with high Curie temperatures. However, the microscopic mechanism of their ferroelectric switching is not fully understood. We explore proton tautomerism in the prototypical case of croconic acid, $\mathrm{C}_{5} \mathrm{O}_{5} \mathrm{H}_{2}$. In order to determine how efficiently ferroelectricity in croconic acid is described in terms of its $\Gamma$-point phonon modes, the minimum energy path between its structural ground states is approximated by projection onto reduced basis sets formed from subsets of these modes. The potential energy curve along the minimum energy path was found to be sensitive to the order of proton transfer, which requires a large subset $(z 8)$ of the modes to be approximated accurately. Our findings suggest rules for the construction of effective Hamiltonians to describe proton transfer ferroelectrics.
\end{abstract}

\section{Introduction}

A ferroelectric is a material that exhibits a spontaneous, switchable electric polarization. Since polarization switching is the crucial function, the microscopic mechanism by which this switching occurs holds important scientific interest. This topic has been researched both experimentally ${ }^{1-4}$ and theoretically ${ }^{5-7}$ over the course of many decades for many systems.

Different theoretical approaches have been used to provide insights into ferroelectrics. Before the development of ab initio methods like density functional theory (DFT), ${ }^{8,9}$ polarization reversal processes were modelled using continuum methods such as the Landau-Ginzburg theory, ${ }^{10}$ from which models for domain nucleation and growth could be derived.,11 More recently, DFT has been used to model the atomic structure of defect-free ferroelectric crystals; ${ }^{12}$ and the atomic structure and properties of domain walls, ${ }^{13}$ which is outside the realm of applicability of continuum models. It has also been used to calculate the parameter values of effective Hamiltonian models ${ }^{14}$ constructed in order to simulate large systems beyond the reach of first-principles methods. ${ }^{15,16}$

The microscopic switching mechanism of organic ferroelectrics such as potassium dihydrogen phosphate and triglycine sulphate is characterised by proton transfer between molecular

\footnotetext{
${ }^{a}$ Department of Materials, Imperial College London, London SW7 2AZ, UK. E-mail: laura.ratcliffo8@imperial.ac.uk, a.walsh@imperial.ac.uk

${ }^{b}$ Department of Materials Science and Engineering, Yonsei University, Seoul 03722, Korea

$\dagger$ Electronic supplementary information (ESI) available. See DOI: 10.1039/ docp04236f
}

units. Croconic acid (CRCA) is another member of this category. ${ }^{17,18}$ The motion of protons parallel and perpendicular to the polar axis, and consequent electronic rearrangement are understood to induce the observed polarization in CRCA. ${ }^{18}$ Like many proton transfer ferroelectrics, however, it is less thermally stable than inorganic ferroelectrics such as lead zirconate titanate and polymer ferroelectrics such as polyvinylidene fluoride. The relative ease of practical experimentation and potential application to future devices might explain why, upon surveying the literature, more progress has been made toward explaining switching mechanisms in the latter types of ferroelectrics ${ }^{19,20}$ than in proton transfer ferroelectrics.

In previous work, the degrees of freedom for an effective Hamiltonian for barium titanate were constructed by projecting the unstable phonon modes of the crystal onto individual unit cells. $^{21}$ This effective Hamiltonian successfully described the phase transition sequence of the material. On the other hand, a similar effective Hamiltonian for the proton transfer ferroelectric potassium dihydrogen phosphate (KDP) used its unstable gamma point mode to derive the degrees of freedom $^{22}$ but significantly overestimated KDP's phase transition temperature and predicted a second order phase transition, rather than the weakly first order transition that is experimentally observed. ${ }^{22}$

In this study, the possibility of describing the ferroelectricity of the proton transfer ferroelectric croconic acid (CRCA) by selecting the correct subset of its phonon modes was considered. The minimum energy path (MEP) for polarization switching in CRCA has been calculated with the nudged elastic band $\operatorname{method}^{23}$ in previous work, ${ }^{24}$ and found to involve pairwise transfer of protons. Any reduced basis of atomic displacements 
that can be used to describe ferroelectricity in CRCA must at least be able to express the MEP. Therefore, the ability of the phonon modes of CRCA to efficiently describe ferroelectricity in CRCA was assessed by using these modes as a basis in which to approximate the MEP.

The following analysis bears similarities with previous work ${ }^{20}$ which decomposed polar distortions of two other proton transfer ferroelectrics 1-cyclobutene-1,2-dicarboxylic acid and 2-phenylmalondialdehyde. Both analyses seek to conceptualise the collective atomic distortions that give rise to ferroelectricity in proton transfer ferroelectrics. On the other hand, the authors of ref. 20 decompose the polar distortions of these materials into three different types of atomic distortion instead of the spectra of the materials' $\Gamma$-point phonon modes.

\section{Methods}

The ground-state crystal structure of CRCA has the $P c a 2_{1}$ space group, which is polar. The initial crystal structure of CRCA was taken from entry 147324 of the Cambridge Crystallographic Data Centre and was obtained from X-ray diffraction. ${ }^{25}$ As a starting point, we linearly interpolate a path between the two orientation states, where the direction of polarization is reversed. The mid-point is a non-polar structure that has the space group $\mathrm{Pbcm}$. Equivalently, it is the minimal supergroup from which the $\mathrm{Pca}_{1}$ structure is obtained through the smallest atomic displacements, i.e. Pbcm-CRCA would be its prototype phase, as defined by Aizu. ${ }^{26}$ We take the $\mathrm{Pbcm}$ structure to approximate the hypothetical paraelectric phase, which CRCA would reach if it did not thermally decompose at $T \approx 450 \mathrm{~K}^{17}$

Firstly, we optimise the crystal structures separately under the constraints of the $P c a 2_{1}$ and $P b c m$ symmetry elements. The total energy and forces were calculated from plane-wave DFT as implemented in the Quantum Espresso ${ }^{27,28}$ and Abinit $^{29}$ software packages. To determine whether dispersion corrections to the exchange-correlation energy functional would give a more accurate description of the potential energy surface, geometry optimizations were performed with two different dispersioncorrected functionals: DFT-D3 ${ }^{30}$ (Abinit's implementation) and vdW-DF2 ${ }^{31}$ (Quantum Espresso's implementation). The normal modes were calculated at the $\Gamma$ point by finite differences with the Phonopy package. ${ }^{32}$ Further computational details are given in the Appendix.

\section{Results}

\subsection{Crystal structure}

The optimised structure of the $P c a 2_{1}$ phase can be compared to the results of neutron scattering experiments. ${ }^{33}$ The unit cell dimensions, $\mathrm{OH} / \mathrm{O} \cdots \mathrm{H}$ bond lengths and angles are presented in Table 1. We distinguish between 'hinge' protons and 'terrace' protons whose associated $\mathrm{OH}$. . O triplets are oriented parallel to the [001] and [110] directions, respectively. The hinge and terrace triplets are indicated in Fig. 1 as $\mathrm{O}(5)-\mathrm{H}(6)-\mathrm{O}(8)$ and $\mathrm{O}(20)-\mathrm{H}(8)-\mathrm{O}(14)$, respectively.

The vdW-DF2 functional gave lattice parameters of a similar overall accuracy to DFT-D3, while uncorrected PBE gave much larger errors for the $a$ and $b$ parameters but a smaller error for the $c$ parameter. This is consistent with earlier results; ${ }^{33}$ dispersion interactions between pleated layers of CRCA binds these layers together.

vdW-DF2 also gave the most accurate bond lengths and angles; however these parameters were also well reproduced by the uncorrected functional. This may be explained by the fact that strong hydrogen bonds (i.e. those possessing donorhydrogen-acceptor angles $\left.(\angle \mathrm{DHA}) \sim 180^{\circ}\right)$ exist between the $\mathrm{C}_{5} \mathrm{O}_{5} \mathrm{H}_{2}$ molecules in CRCA. Such bonds are understood to contain a smaller dispersive element than that of weak hydrogen bonds, which generally have $\angle$ DHA substantially smaller than $180^{\circ}$. $^{34}$

As vdW-DF2 gave the most accurately optimized structure overall, it was used for the subsequent calculations of the

Table 1 Comparison between calculated parameters of the crystal structure of croconic acid relaxed with dispersion corrections (DFT-D3, vdW-DF2) and without. The Hermann-Mauguin symbols refer to the space groups to which the crystal was constrained during unit cell optimisation. The error is given with respect to the result calculated from low temperature neutron diffraction measurements. ${ }^{a}$ The convention adopted in the literature, ${ }^{a}$ which refers to protons of $\mathrm{OH} \cdots \mathrm{O}$ triples oriented parallel to [001] as 'hinge' protons and those of triples oriented parallel to [110] as 'terrace' protons is used. $\theta_{\text {hinge }}$ and $\theta_{\text {terrace }}$ refer to angles of the corresponding triples

\begin{tabular}{|c|c|c|c|c|c|c|c|c|}
\hline & \multicolumn{7}{|l|}{$\underline{\mathrm{Pca}_{1}}$} & \multirow{2}{*}{$\begin{array}{l}\text { Pbcm } \\
\text { vdW-DF2 }\end{array}$} \\
\hline & Result & Error & Result & Error & Result & Error & Expt. $^{a}$ & \\
\hline$a(\mathrm{bohr}, \%)$ & 16.44 & 0.94 & 17.20 & 5.60 & 16.63 & 2.13 & 16.29 & 16.62 \\
\hline$b$ (bohr, \%) & 10.00 & 3.82 & 10.87 & 12.88 & 9.81 & 1.89 & 9.63 & 9.70 \\
\hline$c($ bohr, \%) & 20.44 & 0.89 & 20.62 & 0.03 & 20.98 & 1.71 & 20.63 & 20.32 \\
\hline$l(\mathrm{OH})_{\text {terrace }}(\mathrm{bohr}, \%)$ & 1.95 & 2.83 & 1.94 & 1.93 & 1.92 & 1.02 & 1.90 & 2.31 \\
\hline$l(\mathrm{O} \cdots \mathrm{H})_{\text {terrace }}(\mathrm{bohr}, \%)$ & 2.87 & 5.74 & 2.97 & 2.46 & 3.09 & 1.34 & 3.04 & 2.31 \\
\hline$\theta_{\text {hinge }}$ (deg., deg.) & 167.2 & 0.9 & 167.2 & 0.9 & 167.4 & 0.7 & 168.1 & 169.4 \\
\hline$\theta_{\text {terrace }}$ (deg., deg.) & 174.1 & 1.9 & 172.9 & 0.7 & 172.0 & 0.2 & 172.2 & 180.0 \\
\hline
\end{tabular}

${ }^{a}$ S. Mukhopadhyay et al., Chemical Physics, 2013, 427, 95-100. 


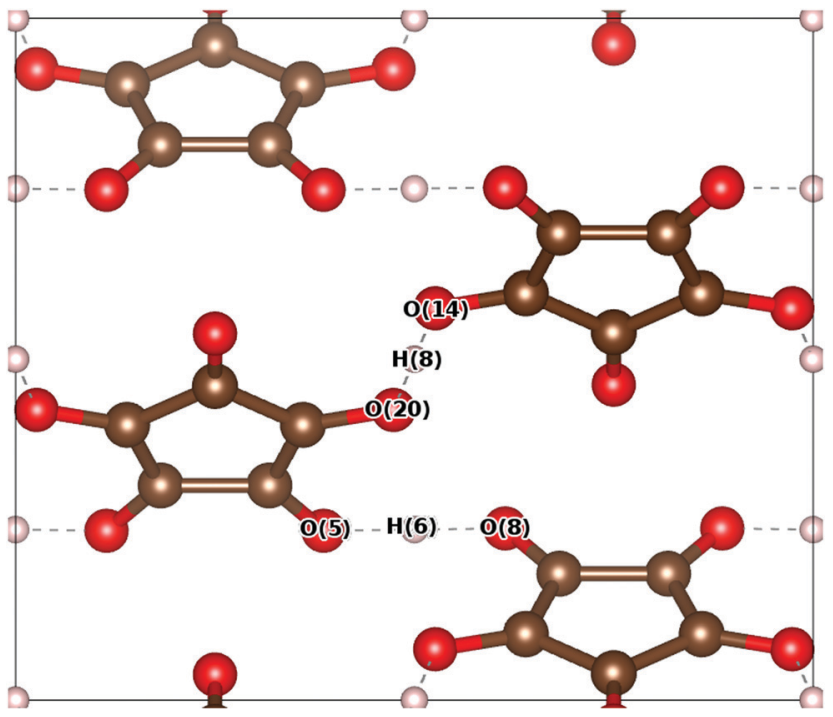

Fig. 1 A view down the a-axis of croconic acid, after Pbcm-constrained relaxation of the atomic positions and cell dimensions. Carbon, oxygen and hydrogen atoms are coloured brown, red and white respectively. The atoms that comprise the terrace $(\mathrm{O}(20), \mathrm{H}(8), \mathrm{O}(14))$ and hinge $(\mathrm{O}(5), \mathrm{H}(6)$, $\mathrm{O}(8)) \mathrm{OH} \ldots \mathrm{O}$ triples are labelled.

dynamical matrix at $\Gamma$ and the minimum energy path. For the calculation of the Born effective charge (BEC) tensor, a structure found by $\mathrm{Pbcm}$ symmetry-constrained relaxation of CRCA with the uncorrected PBE functional was used. The accurate bond lengths and angles predicted by this functional implied that the electronic rearrangement induced by atomic displacement that contributes to the BEC tensor would also be accurately predicted. Further details are given in the Appendix.

\subsection{Vibrational structure}

A complete listing of the normal modes of $\mathrm{Pbcm}$-CRCA, including irreducible representations, is given in the ESI. $\dagger$ As there are $N=48$ atoms in the unit cell, there are $144(3 N)$ phonon branches. Referring to these modes by their associated $\mathrm{Pbcm}$ symmetry labels would be ambiguous; therefore, in the following we refer to each mode by its index when the modes are arranged in order of ascending frequency. Modes 0-6 are imaginary, while modes 7-9 are acoustic (zero frequency) translations. The remaining modes are stable with positive frequencies.

\subsection{The minimum energy path}

The minimum energy path for ferroelectric switching in CRCA has been calculated according to the nudged elastic band algorithm in previous work $^{24}$ which used the same vdW$\mathrm{DF}^{31}$ exchange-correlation functional employed here. This calculation was reproduced in the present work in order to determine how efficiently the $\Gamma$-point phonon modes approximate this path. The result of this calculation is illustrated in Fig. 3. The energy barrier associated with this path is $0.045 \mathrm{eV}$ per proton. The $12 \%$ difference relative to the corresponding value calculated in the prior work ${ }^{24}(0.051 \mathrm{eV}$ per proton $)$ could be due to the different unit cell dimensions used in the present work. Nevertheless, the MEPs calculated in both works involve the transfer of the 8 protons in the unit cell of CRCA in 4 successive pairs.

Approximations to the minimum energy switching path were made by repeatedly projecting it onto a reduced basis comprised of a varying number $\left(N_{\text {modes }}\right)$ of $\Gamma$-point phonon modes. The order in which modes were added to the basis was found by forward-selection so that the most important modes were added first. Specifically, the algorithm involved the following steps. For $N_{\text {modes }}=1, \ldots, 3 N_{\text {atom: }}$ :

1. Calculate the error in approximating the MEP according to

$$
\varepsilon_{\mathrm{MEP}}\left(N_{\text {modes }}\right) \equiv \sqrt{\frac{1}{3 N_{\text {image }} N_{\text {atom }}} \sum_{a n}\left(\mathbf{x}_{a n}\left(N_{\text {modes }}\right)-\mathbf{x}_{a n}(\mathrm{MEP})\right)^{2}}
$$

where $\boldsymbol{x}_{a n}$ is the position of atom $a$ in NEB image $n$ after separately adding each of the remaining modes to the basis.

2. Add the mode which gives the smallest $\varepsilon_{\mathrm{MEP}}$ value, and remove it from the set of remaining modes.

3. If $N_{\text {modes }}<3 N_{\text {atom }}$ increment $N_{\text {modes }}$ by 1 and return to step 1, else terminate.

The energy along approximations to the MEP found by adding modes to the basis in the order described above was evaluated. The results of these calculations are illustrated in Fig. 3 which plots the potential energy against a coordinate, $\lambda$, normalised between -1 and +1 , that quantifies the location of an atomic configuration on a line in $3 N_{\text {atom }}$-dimensional configuration space between the two orientation states. Precisely, $\lambda$ is defined as

$$
\lambda \equiv 2\left(\mathbf{X}-\mathbf{X}^{(-)}\right) \cdot \frac{\Delta \mathbf{X}}{|\Delta \mathbf{X}|}-1
$$

for an atomic configuration $\mathbf{X}$ ) ( $X_{a i}$ is the coordinate $i$ of atom $a$ 's position), and where $\Delta \mathbf{X} \equiv \mathbf{X}^{(+)}-\mathbf{X}^{(-)}$is the atomic displacement between the 'positive' and 'negative' orientation states. After 8 modes were added to the basis, the qualitative shape of the MEP potential energy curve (PEC) was reproduced. Modes that were added subsequently led to a downward shift of the entire PEC as the basis for approximating the MEP became more complete. Adding modes in order of ascending $\omega^{2}$, for example, instead of determining the order of mode inclusion using the method described above would have led to PECs that do not approach the MEP PEC in a continuous way for $N_{\text {modes }} \lesssim 120$. This contrast is illustrated in Fig. 2.

\subsection{Proton transfer extent and the minimum energy path}

An explanation of how the PECs of approximations to the MEP improved for $N_{\text {modes }} \leq 8$ was sought. As described in previous work $^{24}$ and reproduced here, the MEP of ferroelectric switching in CRCA involves successive pairwise transfer of protons. Crucially, the protons of CRCA transfer in a well-defined order which is only accurately reproduced with 8 modes in the basis. This is illustrated in Fig. 4 which plots the mean absolute error 


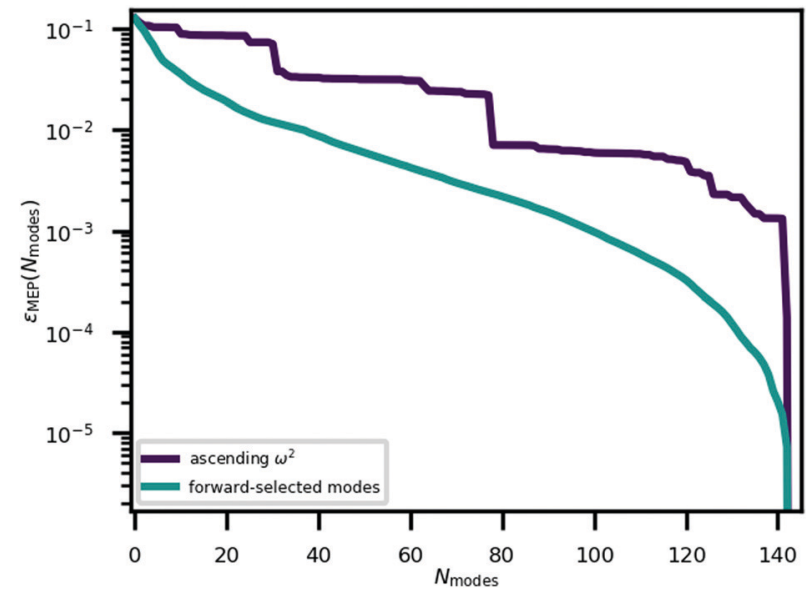

Fig. 2 The error in approximating the minimum energy path for ferroelectric switching with $\Gamma$-point phonon modes added in an order determined by a forward selection procedure described in Section 3.3. The corresponding error found by adding the modes in order of ascending $\omega^{2}$ is added for comparison.

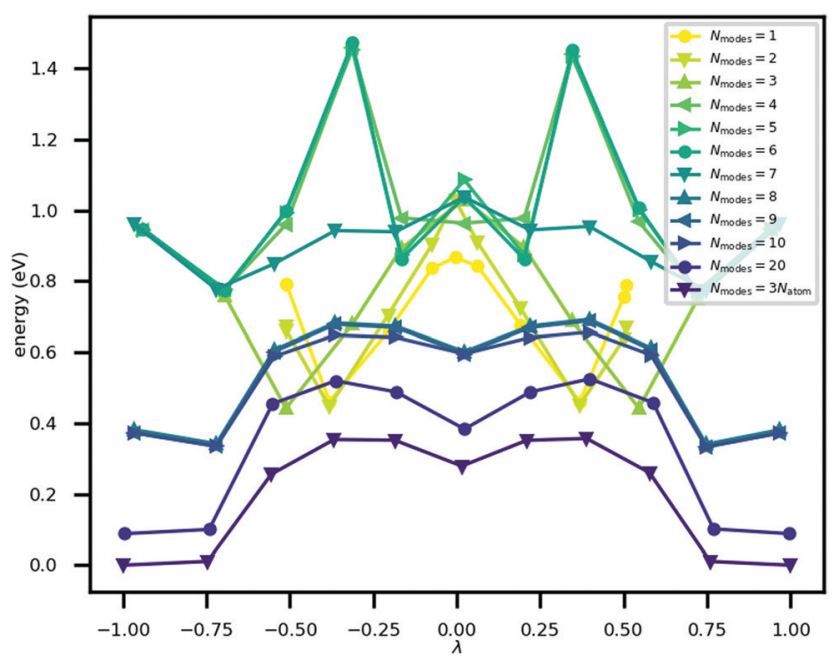

Fig. 3 The potential energy curve along approximations to the minimum energy switching path between states with opposite polarization $(\lambda \pm 1)$. These vary in the number of phonon modes $\left(N_{\text {modes }}\right)$ in the approximation basis of the structural transformation.

in proton transfer extent defined as

$$
\varepsilon_{\mathrm{PTE}}\left(N_{\text {modes }}\right) \equiv \frac{1}{N_{\text {protons }} N_{\text {image }}} \sum_{p n}\left|\delta l_{p}^{(n)}\left(N_{\text {modes }}\right)-\delta l_{p}^{(n)}(\mathrm{MEP})\right|
$$

where

$$
\delta l_{p}^{(n)}\left(N_{\text {modes }}\right) \equiv \frac{l_{\left(O_{\text {donor }} H^{(p)}\right)}^{(n)}\left(N_{\text {modes }}\right)-l_{\left(O_{\text {acceptor }} H^{(p)}\right)}^{(n)}\left(N_{\text {modes }}\right)}{2}
$$

is the extent of transfer of proton $p$ in image $n$ of the MEP when approximated by $N_{\text {modes }}$ modes.

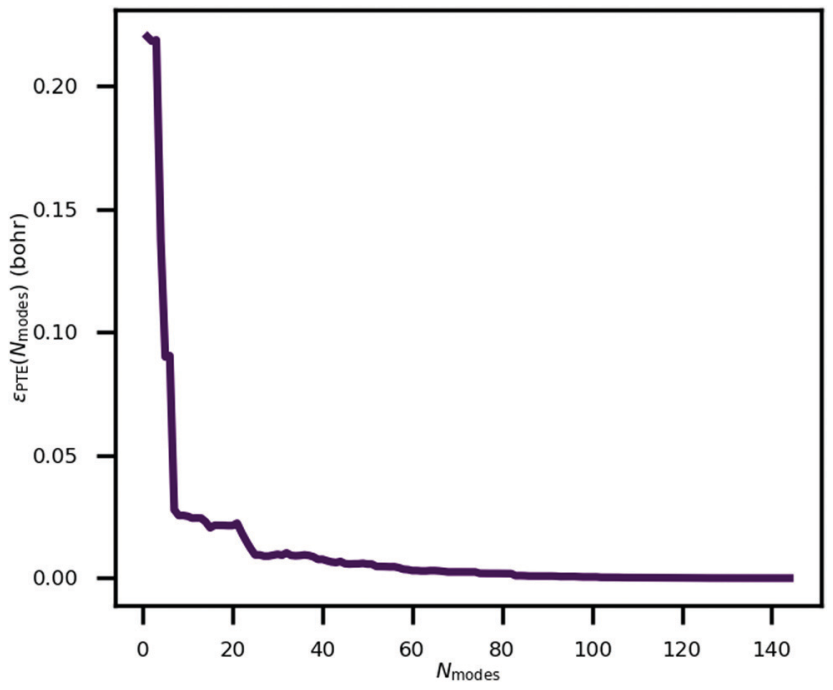

Fig. 4 The mean absolute error in proton transfer extent, as defined in eqn (3), incurred by approximating the minimum energy path for ferroelectric switching in croconic acid by a finite number of phonon modes, $N_{\text {modes. }}$

It can be seen in the figure that between $N_{\text {modes }}=1$ and 8 , $\varepsilon_{\mathrm{PTE}}$ drops significantly by $88 \%$ to a small plateau, at which point the qualitative shape of the MEP PEC is recaptured. The configuration of hydroxyl bond lengths appears to be a critical determinant of the potential energy of the crystal.

Fig. 3 shows that the MEP PEC features a local minimum at its centre; the structure at $\lambda=0$ is metastable. An analysis of the contributions to the potential energy for each image in the MEP suggests that this state is stabilised by electrostatic interactions in the system. This analysis is illustrated in Fig. 5 which plots the electrostatic (Hartree plus ionic) and non-electrostatic contributions to total energy for each MEP image. The figure shows a drop in electrostatic energy at the centre of the MEP

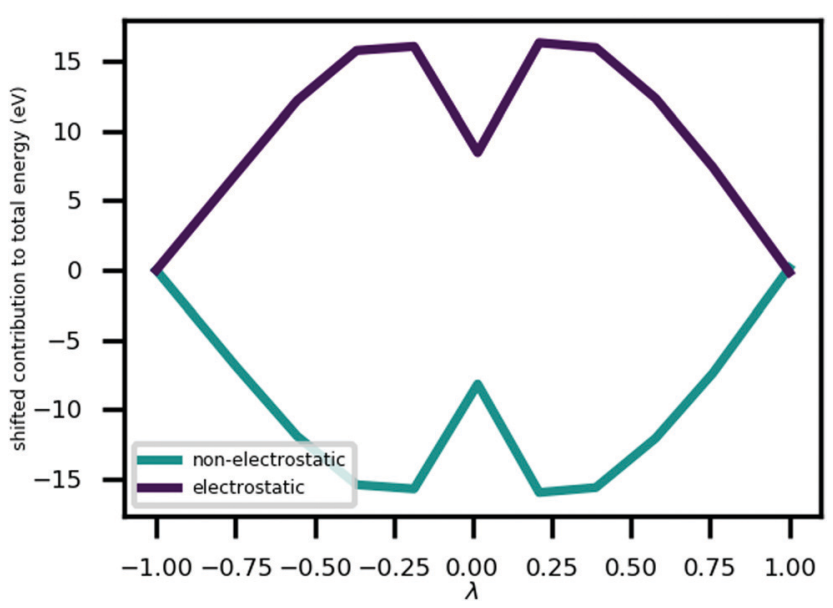

Fig. 5 A plot of the 'electrostatic' (i.e. Hartree energy plus ionic interaction energy) and 'non-electrostatic' (i.e. total energy minus electrostatic contribution) contributions to the total energy at each point on the minimum energy path, relative to their values at $\lambda=-1$. 
Table 2 A representative sample of the Born effective charge tensor of the $\mathrm{H}$ ions in croconic acid. Due to the $\mathrm{Pbcm}$ symmetry of the hypothetical paraelectric phase of CRCA, these values apply to all hinge and terrace protons in the unit cell

\begin{tabular}{llll}
\hline & \multicolumn{3}{l}{ Norm of Born effective charge tensor in direction $(e)$} \\
\cline { 2 - 4 } Ion & $x$ & $y$ & $z$ \\
\hline H (terrace) & 3.10 & 2.15 & 1.58 \\
H (hinge) & 0.28 & 0.40 & 4.02
\end{tabular}

which is evidently greater than the increase in non-electrostatic contribution to the total energy for that image.

The Born effective charge (BEC) tensors of each $\mathrm{H}$ ion in CRCA has a large anomalous component i.e. the proportion of its value beyond the formal charge of the ion. This is shown in Table 2, that reports the norms of the BEC tensor associated with $\mathrm{H}$ ion displacements along the three coordinate axes i.e. $\sqrt{\sum_{i}\left(Z_{i m}^{a}\right)^{2}}$ for ion $a$, displacement direction $m$. The configuration of hydroxyl bond lengths in CRCA, therefore, has a significant effect on the electrostatic energy, stabilising the $\lambda=0$ structure, as well as affecting the coordination of valence electrons in the ions surrounding the hydroxyl bonds.

\section{Conclusion}

The analysis of the phonon modes of a crystal structure can often be used to describe mechanisms of the structural phase transitions for a given material. While this is well established to the specific cases of inorganic perovskite ferroelectrics, this study has demonstrated that the mechanism of field-induced polarization switching in croconic acid - and, likely, in other proton transfer ferroelectrics - is not efficiently expressed in terms of its phonon modes.

As described in Section 1, the successful effective Hamiltonian for barium titanate ${ }^{15}$ constructed its degrees of freedom by projecting the three unstable modes of the crystal onto individual unit cells, and also including strain. We have shown, however, that the shape of the potential energy curve along the minimum energy switching path of CRCA is sensitive to the precise order of proton transfer, and that the proton transfer mechanism requires at least 8 modes to be approximated accurately enough for the resulting potential energy curve to be qualitatively similar in shape to that of the ideal path. If the construction of an effective Hamiltonian similar to ref. 15 were attempted, at least this many modes would need to be projected onto individual unit cells to construct the degrees of freedom, leading to an impractical number of polynomial terms to fit. A basis for atomic displacements that allows individual atoms to move independently, instead of collectively within a unit cell, would be more suitable for the description of ferroelectricity in a proton transfer ferroelectric.

\section{Computational details}

For the ground state DFT calculations, an SCF convergence tolerance of $10^{-10}$ Ry per ion on the total energy was used. A cutoff of 200 Ry and Monkhorst-Pack mesh of $(3,3,2)$ was sufficient to converge the ground state energy within a tolerance of $8.0 \times 10^{-4}$ Ry per ion, and atomic forces to within a tolerance of $4.0 \times 10^{-5}$ Ry per bohr. Ultrasoft pseudopotentials ${ }^{35,36}$ were used to approximate the core electrons. Dispersion-corrected functionals ${ }^{31,37}$ were used as described in Section 3.

In order to compute the non-analytical term correction $^{38}$ to the dynamical matrix at $\Gamma$ the Born effective charge tensor was calculated according to density functional perturbation theory ${ }^{38}$ as implemented in Abinit. Following the structure optimisation, the Kohn-Sham wavefunctions from the $\mathrm{Pbcm}$ structure were recalculated with an SCF convergence condition of the absolute difference between the squared residual of the Kohn-Sham potential at successive SCF iterations falling below a threshold of $10^{-18} \mathrm{Ha}^{2}$. The first order wavefunctions with respect to perturbations of atomic positions were calculated with an SCF convergence tolerance on the squared (effective) potential residual of $10^{-8} \mathrm{Ha}^{2}$. The potential energy surface calculations were supported by custom scripts to generate distorted atomic configurations and post-process the resulting Quantum Espresso output files.

\section{Conflicts of interest}

There are no conflicts to declare.

\section{Acknowledgements}

This work was supported through a studentship in the Centre for Doctoral Training on Theory and Simulation of Materials at Imperial College London funded by the EPSRC (EP/L015579/1). LER acknowledges support from an EPSRC Early Career Research Fellowship (EP/P033253/1) and the Thomas Young Centre under grant number TYC-101. Via our membership of the UK's HEC Materials Chemistry Consortium, which is funded by EPSRC (EP/L000202), this work used the ARCHER UK National Supercomputing Service (http://www.archer.ac. uk). We are grateful to the UK Materials and Molecular Modelling Hub for computational resources, which is partially funded by EPSRC (EP/P020194/1). We would finally like to thank Karin Rabe for insightful discussions and advice.

\section{Notes and references}

1 E. A. Little, Phys. Rev., 1955, 98, 978-984.

2 A. Gruverman, B. J. Rodriguez, C. Dehoff, J. D. Waldrep, A. I. Kingon, R. J. Nemanich and J. S. Cross, Appl. Phys. Lett., 2005, 87, 082902.

3 G. Vizdrik, S. Ducharme, V. M. Fridkin and S. G. Yudin, Phys. Rev. B: Condens. Matter Mater. Phys., 2003, 68, 094113.

4 C. T. Nelson, P. Gao, J. R. Jokisaari, C. Heikes, C. Adamo, A. Melville, S.-H. Baek, C. M. Folkman, B. Winchester, Y. Gu, Y. Liu, K. Zhang, E. Wang, J. Li, L.-Q. Chen, C.-B. Eom, D. G. Schlom and X. Pan, Science, 2011, 334, 968-971.

5 W. J. Merz, Phys. Rev., 1954, 95, 690-698. 
6 V. Y. Shur and E. L. Rumyantsev, Ferroelectrics, 1994, 151, 171-180.

7 X. Xia, Y. Wang, Z. Zhong and G. J. Weng, Proc. R. Soc. A, 2016, 472, 20160468.

8 P. Hohenberg and W. Kohn, Phys. Rev., 1964, 136, B864-B871.

9 W. Kohn and L. J. Sham, Phys. Rev., 1965, 140, A1133-A1138.

10 Structural Phase Transitions I. Landau theory: Advances in Physics: vol. 29, no. 1, https://www.tandfonline.com/doi/ abs/10.1080/00018738000101346.

11 R. C. Miller and G. Weinreich, Phys. Rev., 1960, 117, 1460-1466.

12 R. D. King-Smith and D. Vanderbilt, Phys. Rev. B: Condens. Matter Mater. Phys., 1994, 49, 5828-5844.

13 B. Meyer and D. Vanderbilt, Phys. Rev. B: Condens. Matter Mater. Phys., 2002, 65, 104111.

14 Y.-H. Shin, V. R. Cooper, I. Grinberg and A. M. Rappe, Phys. Rev. B: Condens. Matter Mater. Phys., 2005, 71, 054104.

15 W. Zhong, D. Vanderbilt and K. M. Rabe, Phys. Rev. B: Condens. Matter Mater. Phys., 1995, 52, 6301-6312.

16 Y.-H. Shin, I. Grinberg, I.-W. Chen and A. M. Rappe, Nature, 2007, 449, 881-884.

17 S. Horiuchi, Y. Tokunaga, G. Giovannetti, S. Picozzi, H. Itoh, R. Shimano, R. Kumai and Y. Tokura, Nature, 2010, 463, 789-792.

18 S. Horiuchi, K. Kobayashi, R. Kumai and S. Ishibashi, Nat. Commun., 2017, 8, 14426.

19 N. Setter, D. Damjanovic, L. Eng, G. Fox, S. Gevorgian, S. Hong, A. Kingon, H. Kohlstedt, N. Y. Park, G. B. Stephenson, I. Stolitchnov, A. K. Taganstev, D. V. Taylor, T. Yamada and S. Streiffer, J. Appl. Phys., 2006, 100, 051606.

20 A. Stroppa, D. Di Sante, S. Horiuchi, Y. Tokura, D. Vanderbilt and S. Picozzi, Phys. Rev. B: Condens. Matter Mater. Phys., 2011, 84, 014101.

21 W. Zhong, D. Vanderbilt and K. M. Rabe, Phys. Rev. Lett., 1994, 73, 1861-1864.

22 G. Colizzi, PhD thesis, Queen's University Belfast, 2005.

23 H. Jónnson, G. Mills and K. W. Jacobsen, Classical and Quantum Dynamics in Condensed Phase Simulations, World Scientific, 1998, ch.16, pp. 385-404.

24 S. Mukhopadhyay, M. J. Gutmann, M. Jiménez-Ruiz, D. B. Jochym, K. T. Wikfeldt, K. Refson and F. FernandezAlonso, Phys. Chem. Chem. Phys., 2017, 19, 32216-32225.

25 D. Braga, L. Maini and F. Grepioni, CrystEngComm, 2001, 3, 27-29.

26 K. Aizu, Phys. Rev. B: Solid State, 1970, 2, 754-772.

27 P. Giannozzi, S. Baroni, N. Bonini, M. Calandra, R. Car, C. Cavazzoni, D. Ceresoli, G. L. Chiarotti, M. Cococcioni, I. Dabo, A. Dal Corso, S. de Gironcoli, S. Fabris, G. Fratesi,
R. Gebauer, U. Gerstmann, C. Gougoussis, A. Kokalj, M. Lazzeri, L. Martin-Samos, N. Marzari, F. Mauri, R. Mazzarello, S. Paolini, A. Pasquarello, L. Paulatto, C. Sbraccia, S. Scandolo, G. Sclauzero, A. P. Seitsonen, A. Smogunov, P. Umari and R. M. Wentzcovitch, J. Phys.: Condens. Matter, 2009, 21, 395502.

28 P. Giannozzi, O. Andreussi, T. Brumme, O. Bunau, M. B. Nardelli, M. Calandra, R. Car, C. Cavazzoni, D. Ceresoli, M. Cococcioni, N. Colonna, I. Carnimeo, A. D. Corso, S. de Gironcoli, P. Delugas, R. A. D. Jr, A. Ferretti, A. Floris, G. Fratesi, G. Fugallo, R. Gebauer, U. Gerstmann, F. Giustino, T. Gorni, J. Jia, M. Kawamura, H.-Y. Ko, A. Kokalj, E. Küçükbenli, M. Lazzeri, M. Marsili, N. Marzari, F. Mauri, N. L. Nguyen, H.-V. Nguyen, A. O. de-la Roza, L. Paulatto, S. Poncé, D. Rocca, R. Sabatini, B. Santra, M. Schlipf, A. P. Seitsonen, A. Smogunov, I. Timrov, T. Thonhauser, P. Umari, N. Vast, X. Wu and S. Baroni, J. Phys.: Condens. Matter, 2017, 29, 465901.

29 X. Gonze, F. Jollet, F. Abreu Araujo, D. Adams, B. Amadon,

T. Applencourt, C. Audouze, J. M. Beuken, J. Bieder, A. Bokhanchuk, E. Bousquet, F. Bruneval, D. Caliste, M. Côté, F. Dahm, F. Da Pieve, M. Delaveau, M. Di Gennaro, B. Dorado, C. Espejo, G. Geneste, L. Genovese, A. Gerossier, M. Giantomassi, Y. Gillet, D. R. Hamann, L. He, G. Jomard, J. Laflamme Janssen, S. Le Roux, A. Levitt, A. Lherbier, F. Liu, I. Lukačević, A. Martin, C. Martins, M. J. T. Oliveira, S. Poncé, Y. Pouillon, T. Rangel, G. M. Rignanese, A. H. Romero, B. Rousseau, O. Rubel, A. A. Shukri, M. Stankovski, M. Torrent, M. J. Van Setten, B. Van Troeye, M. J. Verstraete, D. Waroquiers, J. Wiktor, B. Xu, A. Zhou and J. W. Zwanziger, Comput. Phys. Commun., 2016, 205, 106-131.

30 S. Grimme, J. Antony, S. Ehrlich and H. Krieg, J. Chem. Phys., 2010, 132, 154104.

31 K. Lee, É. D. Murray, L. Kong, B. I. Lundqvist and D. C. Langreth, Phys. Rev. B: Condens. Matter Mater. Phys, 2010, 82, 081101.

32 A. Togo and I. Tanaka, Scr. Mater., 2015, 108, 1-5.

33 S. Mukhopadhyay, M. J. Gutmann, M. Jura, D. B. Jochym, M. Jimenez-Ruiz, S. Sturniolo, K. Refson and F. FernandezAlonso, Chem. Phys., 2013, 427, 95-100.

34 J. Ireta, J. Neugebauer and M. Scheffler, J. Phys. Chem. A, 2004, 108, 5692-5698.

35 D. Vanderbilt, Phys. Rev. B: Condens. Matter Mater. Phys., 1990, 41, 7892-7895.

36 A. Dal Corso, Comput. Mater. Sci., 2014, 95, 337-350.

37 S. Grimme, J. Comput. Chem., 2006, 27, 1787-1799.

38 X. Gonze and C. Lee, Phys. Rev. B: Condens. Matter Mater. Phys., 1997, 55, 10355-10368. 